IMMUNOLOGICAL ASPECTS

\title{
Mycobacterium tuberculosis-induced neutrophil ectosomes decrease macrophage activation
}

\author{
Tonya Azevedo Duarte ${ }^{\mathrm{a}, \mathrm{b}, *}$, Alberto Augusto Noronha-Dutra ${ }^{\mathrm{c}, \mathrm{d}}$, Joilda Silva Nery ${ }^{\mathrm{d}}$, \\ Samantha Brum Ribeiro ${ }^{b}$, Thassila Nogueira Pitanga ${ }^{d}$, José R. Lapa e Silva ${ }^{b}$, Sérgio Arruda ${ }^{d}$, \\ Neio Boéchat ${ }^{b}$ \\ ${ }^{\text {a } H e a l t h ~ S c i e n c e ~ I n s t i t u t e, ~ F e d e r a l ~ U n i v e r s i t y ~ o f ~ B a h i a, ~ S a l v a d o r, ~ B A, ~ B r a z i l ~}$

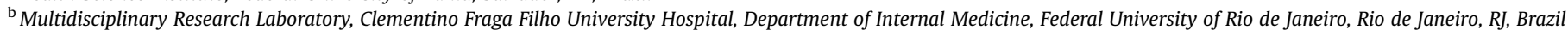 \\ ${ }^{\mathrm{c}}$ University College London, Gower Street, London, UK \\ ${ }^{\mathrm{d}}$ Gonçalo Moniz Research Center, FIOCRUZ, Salvador, BA, Brazil
}

\section{A R T I C L E I N F O}

\section{Article history:}

Received 14 September 2011

Received in revised form

9 February 2012

Accepted 11 February 2012

\section{Keywords:}

Cellular immune response

Tuberculosis

Human polymorphonuclear neutrophil

Ectosomes

Mycobacterium tuberculosis

\begin{abstract}
S U M M A R Y
Background: The existence of ectosome-like microvesicles released by neutrophils was proposed a few decades ago. Other studies revealed that the innate immune response during mycobacterial infection is accompanied by an intense migration of neutrophils to the site of infection, which may be important during the acute phase of tuberculosis. We found that the ectosomes derived from infected neutrophils are biologically active and can influence the survival of Mycobacterium tuberculosis within macrophages. Methods: Mycobacteria were cultured on supplemented Middlebrook-7H9 broth. All strains were grown to the exponential phase and quantitated by serial dilution. Human neutrophils and macrophages were infected with mycobacteria. Ectosomes from neutrophils were isolated post-infection and characterized by transmission electron microscopy and flow cytometry. To determine whether these microvesicles influenced mycobactericidal activity, mycobacteria-infected macrophages were treated with isolated ectosomes.

Results: Ectosomes were released from neutrophils infected with mycobacteria. These ectosomes were derived from neutrophil plasma membrane and a small proportion stained with PKH26. These microvesicles, when incubated with infected macrophages, influenced antimycobacterial activity.

Conclusions: This is the first study to demonstrate that ectosomes that are shed from infected neutrophils influence mycobactericidal activity in macrophages in vitro, suggesting that these microvesicles have biological significance. Nevertheless, major gaps in our knowledge of microvesicle biology remain.
\end{abstract}

(c) 2012 Elsevier Ltd. All rights reserved.

\section{Introduction}

Following infection with Mycobacterium tuberculosis (MTB), most immunocompetent adults are able to develop an immune response that controls mycobacterial proliferation. Although these individuals continue to harbor small numbers of viable mycobacteria, clinical signs of tuberculosis (TB) do not develop. ${ }^{1}$ Pathogenic

\footnotetext{
* Corresponding author. Rua Waldemar Falcão, 121, Candeal, CEP 40296-710, Salvador, BA, Brazil. Tel.: +55 713176 2232; +55 7188035040 (mobile); fax: +55 7131762327.

E-mail addresses: tonya.duarte@gmail.com (T.A. Duarte), adutra@ pqvisitante.bahia.fiocruz.br (A.A. Noronha-Dutra), joilda_nery@yahoo.com.br (J.S. Nery), sbrmicroimuno@yahoo.com.br (S.B. Ribeiro), pitanga.tn@hotmail.com (T.N. Pitanga), jrlapa.ntg@terra.com.br (J.R. Lapa e Silva), arrudasm@gmail.com (S. Arruda), n_boechat@yahoo.com (N. Boéchat).
}

mycobacteria survive and proliferate within mononuclear phagocytes, cells that are normally capable of eliminating most internalized organisms. ${ }^{2}$ The ability of the host to mount an effective immune and inflammatory response that results in increased mycobactericidal activity in macrophages is clearly important for controlling MTB infection. However, it is largely accepted that the optimal anti-MTB response should also restrain the inflammatory process, as excessive inflammation may lead to increased tissue damage and will contribute to TB active disease development. ${ }^{3}$ Considerable evidence indicates that antigen-specific T-cells, activated macrophages, dendritic cells and cytotoxic T-cells are essential for containing MTB infection. Other immune and nonimmune cells may also play a significant role during the host response against mycobacterial infections. ${ }^{4,5}$ Several studies, both experimental and clinical, have shown that acute pulmonary tuberculosis is accompanied by an influx of polymorphonuclear 
neutrophils (PMN) ${ }^{6-11}$ Neutrophils migrate from the blood to the tissues and eventually ingest and kill invading pathogens, including MTB, by generating reactive oxygen intermediates together with other potent antimicrobial compounds released during the process of degranulation. ${ }^{9}$ Human PMN have the capacity to recruit additional PMN along with other immune cells to inflammatory sites by producing and releasing chemokines and cytokines. ${ }^{12-16}$ Several in vitro studies suggest that the impaired ability of PMN from TB patients to generate an effective oxidative burst may contribute to the establishment and spread of MTB infection., 9,17,18 Interestingly, activated PMN release microvesicles directly from the cell surface membrane. ${ }^{19}$ Several different names have been used to describe these small vesicles, including microvesicles, microparticles and ectosomes, probably because the mechanism involved in their release is not known in detail. ${ }^{20-24}$ These neutrophil-derived ectosomes are generated either spontaneously or in response to various stimuli, ${ }^{20-22,24,25}$ and they can differ at the molecular level, depending on the trigger stimuli. ${ }^{26}$ Importantly, the ectosomes are not just by-products of cell activation because they have been shown to have functional activity and can modulate other cells. $^{20,27-29}$ Ectosomes were recently shown to only be secreted by living cells and they are clearly distinct from the microvesicles produced by apoptotic cells. ${ }^{27}$ In addition to their generation kinetics, ectosomes and apoptotic bodies differ in composition and size, with apoptotic bodies being many times larger in diameter and volume. ${ }^{19,23}$ Several studies suggest that the shedding of ectosomes is an important mechanism by which PMN discharge active molecules present in their plasma membranes. ${ }^{11,23,26,30}$ This phenomenon may result in decreased cell surface signaling activity. ${ }^{31}$ During the early stages of inflammation, the ectosomes shed by neutrophils stimulate the release of anti-inflammatory molecules such as transforming growth factor (TGF)- $\beta$ and interleukin (IL)-10 from macrophages while reducing the production of tumor necrosis factor (TNF)- $\alpha$ and IL-8. ${ }^{11,27}$

The remarkable influx and continued presence of both activated phagocyte types in the lungs during MTB infection ${ }^{32,33}$ indicate that PMN might interact with macrophages, thereby interfering with inflammatory and antimycobacterial activity. In the present study, we demonstrate that in vitro MTB infection of human PMN induced the generation of PMN ectosomes. We also describe the morphological features of these ectosomes with transmission electron microscopy imaging and investigate their effect on the antibacterial activity of human macrophages against virulent MTB.

\section{Materials and methods}

\subsection{M. tuberculosis complex strains}

M. tuberculosis (H37Rv - American Type Culture Collection ATCC 25618, New York, NY, USA) and Mycobacterium bovis (ATCC 19274, Rockville, MD, USA) strains were kindly provided by the National Institute of Quality Health Control (INCQS-FIOCRUZ, RJ, BR). Bacteria were rehydrated in Middlebrook-7H9 broth (Difco, MI, USA) supplemented with $10 \%$ albumin dextrose concentrate (ADC - BD BBL - Sparks, MD, USA) and maintained at $37{ }^{\circ} \mathrm{C}$ in an aerobic atmosphere for 15 days. After incubation, homogeneous cell suspensions were obtained by mild sonication using a Vibra-Cell Ultrasonic Processor (Sonics \& Materials Inc., CT, USA) to disaggregate the clumps. The total number of mycobacteria in the suspension was ascertained by enumeration of colony forming units (CFU) in Middlebrook-7H10 broth (Difco, MI, USA) supplemented with $10 \%$ oleic acid albumin dextrose concentrate (OADC - BD BBL - Sparks, MD, USA) maintained at $37{ }^{\circ} \mathrm{C}$ in an aerobic atmosphere for 45 days. All of the stocks were quantified by serial dilution followed by CFU enumeration. Aliquots were stored in $20 \%$ glycerol at $-80^{\circ} \mathrm{C}$ until use.

\subsection{Purification of PMN}

Heparinized blood was obtained from healthy human volunteers. Approximately $20 \mathrm{~mL}$ of venous blood was gently mixed with 3\% Dextran T500 (Sigma, MO, USA) and left for $30 \mathrm{~min}$ for erythrocyte sedimentation. The leukocyte-rich supernatant was aspirated and centrifuged at $200 \mathrm{~g}$ for $10 \mathrm{~min}$ at $24^{\circ} \mathrm{C}$. The pellet was resuspended in $12 \mathrm{~mL}$ of Hanks balanced salt solution (HBSS) in the absence $\mathrm{Ca}^{2+} / \mathrm{Mg}^{2+}$. This suspension was layered over $6 \mathrm{~mL}$ of Histopaque-1077 (Sigma, MO, USA) and centrifuged at $350 \mathrm{~g}$ for $20 \mathrm{~min}$ at $24{ }^{\circ} \mathrm{C}$. After removal of the supernatant, PMN were recovered from the bottom of the tube. The pellet was resuspended in $2 \mathrm{~mL}$ of PBS and $5 \mathrm{~mL}$ of ultra-pure water was added to hypotonic lyses residual erythrocytes. After $1 \mathrm{~min}$, isotonicity was restored by adding $2 \mathrm{~mL}$ of $5 \% \mathrm{NaCl}$. The cells were then centrifuged at $350 \mathrm{~g}$ for $10 \mathrm{~min}$ at $4{ }^{\circ} \mathrm{C}$ and resuspended in $10 \mathrm{~mL}$ of HBSS without $\mathrm{Ca}^{2+}$ / $\mathrm{Mg}^{2+}$. The number of PMN was calculated by counting in a Neubauer chamber. PMN ( $10^{6}$ cells $\left./ \mathrm{mL}\right)$ were then diluted in RPMI1640 medium (LGC, Biotechnology, SP, BR) supplemented with $10 \%$ heat-inactivated fetal calf serum - FCS (LGC, Biotechnology, SP, BR), $2 \mathrm{mM}$ L-glutamine and $2 \mathrm{~g} / \mathrm{L}$ sodium bicarbonate. PMN purity was determined on the basis of nuclear morphology using light microscopy and the level of viability was $95 \%$ at the time of stimulation, as determined by Trypan Blue exclusion technique (Gibco, NY, USA).

\subsection{Isolation of ectosomes from infected PMN}

For stimulation with mycobacteria, PMN were diluted at $10^{7}$ cell $/ \mathrm{mL}$ in previously warmed RPMI-1640, exposed to M. tuberculosis or M. bovis $\left(10^{7}\right.$ bacilli $\left./ \mathrm{ml}\right)$ and incubated for $2 \mathrm{~h}$ at $37{ }^{\circ} \mathrm{C}$ in $5 \% \mathrm{CO}_{2}$. In all cases, the supernatants were collected and centrifuged twice at $4000 \mathrm{~g}$ for $10 \mathrm{~min}$ prior to further ultracentrifugation at $100,000 \mathrm{~g}$ for $1 \mathrm{~h}$ at $4{ }^{\circ} \mathrm{C}$. Ectosome pellets were resuspended in $\mathrm{PBS}$ and stored in aliquots at $-80{ }^{\circ} \mathrm{C}$ prior to use.

\subsection{Ultrastructural analysis of ectosomes by transmission electron microscopy (TEM)}

Ectosomes isolated as described above were resuspended and fixed in a $0.1 \mathrm{mM}$ cacodylate buffer containing $2 \%$ glutaraldehyde. They were then post-fixed for $1 \mathrm{~h}$ at room temperature with $1 \%$ osmium tetroxide in $0.1 \mathrm{mM}$ cacodylate buffer. Next, they were concentrated in a $2 \%$ agar in cacodylate buffer. The samples were dehydrated in a graded series of acetone solutions and embedded in Polybed resin. To visualize the ectosomes, thin sections were sequentially stained with $2 \%$ uranyl acetate in methanol and lead citrate and examined with a Zeiss EM 109 transmission electron microscope at $80 \mathrm{Kv}$ (München, Germany). Ectosomes were defined as round-shaped membrane vesicles, homogenous in size and not exceeding $300 \mathrm{~nm}$ in diameter. ${ }^{22,30}$ Membrane debris was defined as heterogeneous membrane fragments of variable shapes and sizes.

\subsection{Fluorescence-activated cell sorting of ectosomes and apoptotic cells}

The fluorescence-activated cell sorting (FACS) analytical conditions were standardized, e.g. settings of the flow cytometry and quality of the reagents. A first series of experiments using standard silica beads of $1000 \mathrm{~nm}$ diameter (Corpuscular Inc., NY, USA) were run to calibrate the size and size distribution of PMN-derived ectosomes. The resulting data served as an internal control for all our ectosome-related FACS experiments. The forward scatter (FSC) and side scatter (SSC) parameters were plotted on logarithmic 
scales to appropriately cover a wide size range. Microparticles presenting a diameter between 50 and $300 \mathrm{~nm}$ were considered as ectosomes.

To label membranes of isolated ectosomes for FACS analysis, a fluorescent cell linker dye (PKH26, Sigma, MO, USA) was used, following the labeling procedure provided by the manufacturer. The excitation and emission peaks for PKH26 are $551 \mathrm{~nm}$ and $567 \mathrm{~nm}$, respectively. Activated neutrophils were briefly washed twice with PBS and resuspended at a concentration of $2 \times 10^{7}$ cells/ $\mathrm{mL}$ in diluent $\mathrm{C}$. The suspension was mixed with $1 \mathrm{~mL}$ of diluent $\mathrm{C} /$ dye solution (dye diluted $1 / 200$ ) for 2-5 min at room temperature with gentle shaking. The staining reaction was stopped by adding an equal volume of FCS for $1 \mathrm{~min}$ at $22^{\circ} \mathrm{C}$. The sample was diluted with an equal volume of RPMI-1640 and pelleted by centrifugation for $10 \mathrm{~min}$ at $400 \mathrm{~g}$ to remove the staining solution. Ectosomes were obtained from PKH26-stained PMN by a two-step procedure involving an initial centrifugation at $4000 \mathrm{~g}$ for $10 \mathrm{~min}$ to remove cells, followed by ultracentrifugation of the supernatant at $100,000 \mathrm{~g}$ for $1 \mathrm{~h}$ at $4{ }^{\circ} \mathrm{C}$. Unstained neutrophil-released ectosomes were analyzed under the same conditions for establishment of true negative controls. All reagents and solutions used for the ectosome analysis were sterile and filtered ( $0.2 \mu \mathrm{m}$ filter).

The samples were analyzed with a FACSort Cell Sorter flow cytometer. The FSC and fluorescence channel (FL-2) were set on logarithmic scales. An acquisition threshold was set based on FSC to reduce the background signal.

Apoptotic cells were quantified by staining with annexin $\mathrm{V}$ and propidium iodide following the manufacturer's instructions (annexin V-FITC apoptosis detection kit - Sigma, MO, USA). Briefly, the cells were washed with binding buffer and stained with annexin $\mathrm{V}$ and propidium iodide for $10 \mathrm{~min}$ at room temperature and protected from light. FACS analysis was conducted using a FACSort Cell Sorter flow cytometer (BD Bioscience, CA, USA).

\subsection{Isolation and culture of human monocyte-derived macrophages (HMDM)}

Peripheral blood mononuclear cells were isolated from the buffy coat of healthy donors (provided, with permission, by the Fundação de Hematologia e Hemoterapia da Bahia - HEMOBA) by differential centrifugation, first over Histopaque-1077 and then on Percoll (LGC Biotechnology, SP, BR) gradients. The monocyte fraction was plated in 24-well plates (Tissue Culture Plate, 24 well, Becton Dickinson Labware, NJ, USA) at $10^{6}$ cells/1000 $\mu$ L/well containing RPMI-1640 medium supplemented with $10 \%$ heat-inactivated FCS, $2 \mathrm{mM} \mathrm{L}-$ glutamine, $2 \mathrm{~g} / \mathrm{L}$ sodium bicarbonate and antibiotics (100 UL/mL penicillin and $100 \mu \mathrm{L} / \mathrm{mL}$ streptomycin). Monocyte viability was verified by Trypan Blue exclusion technique. Cell viability at this point was greater than $95 \%$ in all experiments. Adherent cells were incubated at $37{ }^{\circ} \mathrm{C}, 5 \% \mathrm{CO}_{2}$ for 10 days prior to infection with mycobacteria. After 10 days in culture, monocyte morphology was analyzed by light microscopy under $400 \times$ magnification (Nikon Eclipse TS 100, TYO, JP) and the cells became larger, more granular and more clustered, forming small colonies. These features were consistent with the maturation of monocytes to macrophages after 10 days of culture.

\subsection{Infection of human macrophages with M. tuberculosis}

Cryogenic vials containing MTB suspension were defrosted at $4{ }^{\circ} \mathrm{C}$ for $18 \mathrm{~h}$, followed by reactivation in Middlebrook-7H9 broth supplemented $10 \%$ ADC for $4 \mathrm{~h}$ at $37{ }^{\circ} \mathrm{C}$ in an aerobic atmosphere. To infect macrophages, culture medium was removed and $100 \mu \mathrm{L}$ of the mycobacterial suspension were added ( $10^{6}$ mycobacteria/well), followed by the addition of RPMI-1640 medium supplemented with $10 \%$ heat-inactivated FCS, $2 \mathrm{mM}$ L-glutamine, $2 \mathrm{~g} / \mathrm{L}$ sodium bicarbonate and antibiotics (100 UL/mL penicillin and $100 \mu \mathrm{L} / \mathrm{mL}$ streptomycin) to a final volume of $1000 \mu \mathrm{L} /$ well. All macrophages were infected at a multiplicity of infection (MOI) of 1:1 in triplicate with M. tuberculosis (H37Rv). After $2 \mathrm{~h}$, the cells were gently washed with warmed medium to remove extracellular bacilli. The 24-well plates containing the infected macrophages were then incubated at $37^{\circ} \mathrm{C}$ and $5 \% \mathrm{CO}_{2}$ for $96 \mathrm{~h}$, after which $\mathrm{CFU}$ counts were performed. For some experiments, wells were also treated with a suspension containing PMN-derived ectosomes. On the fourth day of infection, the medium was removed from each well and the bacilli were used immediately for enumeration.

\subsection{Ectosome vesicle treatment of human macrophages infected with M. tuberculosis}

HMDM were obtained, cultured in 24-well plates and infected with MTB, as described above. One hour after infection, HMDM were treated with purified ectosomes induced by MTB infection of human PMN. At two time points, 0 and $96 \mathrm{~h}$ post-infection, HMDM were permeabilized and the colony forming units of mycobacteria present in each well were counted.

\subsection{Enumeration of colony forming units (CFU)}

CFU quantification was performed according to the technique previously described by Boechat et al. ${ }^{2}$ with some modifications. Briefly, after removing $175 \mu \mathrm{L}$ of medium from each well, cells were permeabilized with $10 \mu \mathrm{L}$ Middlebrook-7H9 broth containing $10 \%$ saponin (Sigma, MO, USA) for 3 min. After permeabilization, $175 \mu \mathrm{L}$ Middlebrook-7H9 broth supplemented with 10\% ADC was added and the contents of each well were dispersed by vigorous pipetting. Three serial 10-fold dilutions were prepared using Middlebrook7H9 broth and $10 \mu \mathrm{L}$ aliquots were plated in triplicate on Petri dishes containing Middlebrook-7H10 broth supplemented with $10 \%$ OADC. The plates were incubated at $37{ }^{\circ} \mathrm{C}$ for 3 weeks before colonies were counted. Petri dishes were incubated at $37{ }^{\circ} \mathrm{C}$ in an aerobic atmosphere for 45 days. The results are expressed as CFU/ well and, in all experiments, were the average of triplicate values for each experimental condition.

\subsection{Statistical methods}

All results are expressed as means \pm standard error of the mean (SEM) unless otherwise indicated. Comparisons between groups were made by one-way analysis of variance (ANOVA), followed by a Dunnett's post-test with comparison to the untreated or control group. Analyses were performed using the GraphPad Prism 5.0 Software (San Diego, CA). Differences with $P \leq 0.05$ were considered significant.

\section{Results}

\subsection{Morphological characterization and PKH26 labeling of ectosomes isolated from infected PMN}

The ectosomes isolated from PMN 2 h after infection with MTB were clearly identified by TEM as round-shaped membrane microvesicles with varying diameters, from 50 to $200 \mathrm{~nm}$ (Figure 1, panels $\mathrm{A}$ and $\mathrm{B}$ ). The ectosome preparations did not contain any major impurities (Figure 1, panel C).

The PMN-derived microparticle preparations were defined by FACS analysis by size $<1000 \mathrm{~nm}$ (Figure 2, panel A). The ectosomes were localized within the R1 and R2 regions, which encompassed particles with diameters ranging from 50 to $300 \mathrm{~nm}$ (Figure 2, 

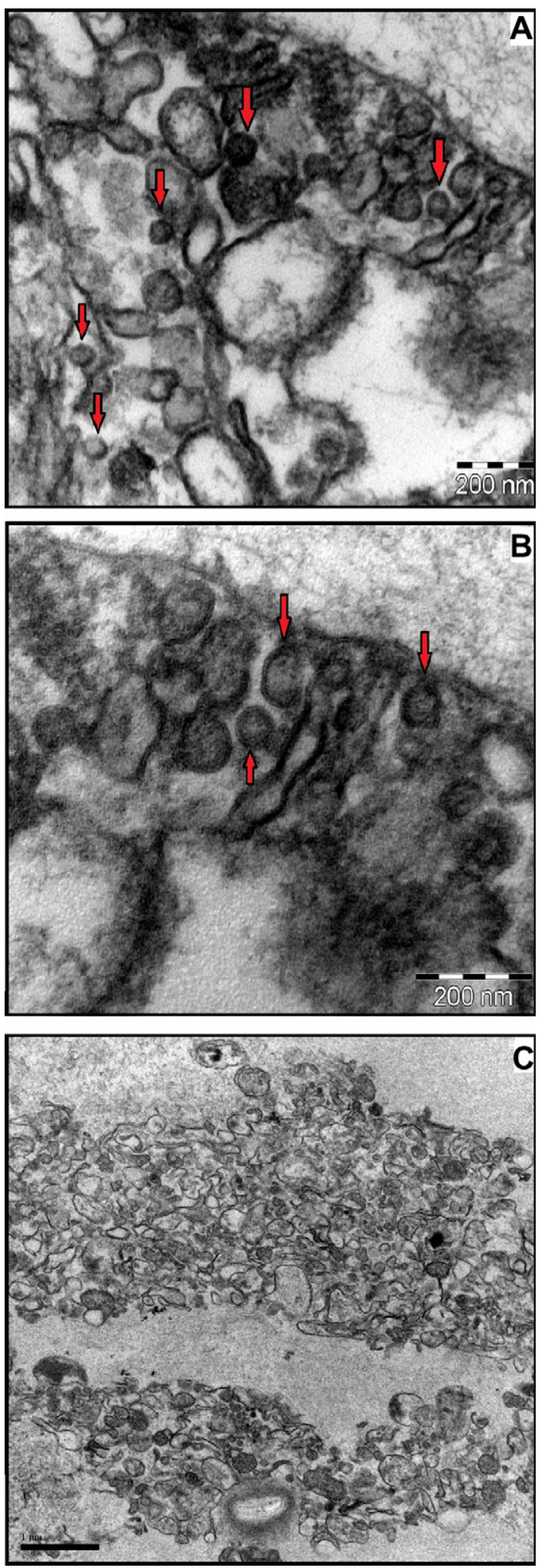

Figure 1. Transmission electron microscopy of purified ectosomes isolated from PMN MTB-infected PMN and released microvesicles, termed ectosomes (arrows), were processed for TEM analysis $2 \mathrm{~h}$ after infection. All ectosomes were less than $200 \mathrm{~nm}$. Scale bar: $0.2 \mu \mathrm{m}$ (panels A and B); $1 \mu \mathrm{m}$ (panel C).

panels B and C). Of note, only the minor microvesicle subpopulation depicted in the R1 region stained positively for PKH26. This staining pattern was observed in ectosome preparations from both infected (8.70\%) and non-infected (8.33\%) PMN (Figure 2, panels D and E).

\subsection{Apoptosis of infected PMN}

To rule out the possibility that the vesicles released from activated neutrophils were apoptotic bodies, we investigated whether $M$. bovis infection triggered early spontaneous apoptosis in PMN.

Control and $M$. bovis-infected neutrophils were stained with annexin $\mathrm{V}(\mathrm{AV})$ and propidium iodide (PI). The translocation of phosphatidylserine (PS) to the outer leaflet of the cell membrane, which can be specifically detected with $\mathrm{AV}$, is one of the early events during the apoptotic process. ${ }^{34}$ PI labels the cellular DNA in necrotic cells in which the cell membrane has been fully compromised. ${ }^{34}$ This combination allows differentiation of early apoptotic cells $\left(\mathrm{AV}^{+} / \mathrm{PI}^{-}\right)$, necrotic cells $\left(\mathrm{AV}^{-} / \mathrm{PI}^{+}\right.$or $\left.\mathrm{AV}^{+} / \mathrm{PI}^{+}\right)$and viable cells $\left(\mathrm{AV}^{-} / \mathrm{PI}^{-}\right)$. Following $2 \mathrm{~h}$ of infection, there was a significant decrease in the percentage of apoptotic and necrotic PMN, represented by the $\mathrm{AV}^{-}$and $\mathrm{PI}^{-}$events in their respective quadrants (Figure 3).

\subsection{Effect of ectosomes on antimycobacterial activity of human macrophages}

Macrophages can acquire neutrophil granules, impacting their antimycobacterial activity. ${ }^{35}$ To investigate whether ectosomes released from viable PMN also interfere with the antimycobacterial activity of macrophages, MTB-infected macrophages were incubated with PMN-derived ectosomes for $96 \mathrm{~h}$ and, after treatment, the CFU number was determined. The addition of ectosomes reduced the antimycobacterial activity of macrophages (Figure 4). Ectosomes were derived from uninfected and MTB-infected neutrophils. Both ectosome preparations similarly inhibited the antimycobacterial activity of macrophages.

A minimal CFU number was measured when the experiments were conducted only with MTB and ectosomes, indicating that few if any bacilli were present in the ectosome preparation released from MTB-infected neutrophils. These data indicate that ectosomes provide a vehicle for regulating the antimicrobial activity of macrophages infected with intracellular mycobacteria.

\section{Discussion}

In response to an infectious challenge, neutrophils are among the first innate immune cells to migrate from the blood to the sites of microbial entry. Protective immunity against MTB has been traditionally ascribed to T-cells and macrophages. ${ }^{1}$ However, the influx of PMN may also play a role in the innate immune response, especially during the initial phases of MTB infection. ${ }^{36}$ Neutrophils secrete chemokines, such as CXCL- 8 , IP-10, CCL- 2 , MIP- $1 \alpha / \beta,{ }^{12-15}$ and pro-inflammatory cytokines including IFN- $\gamma$ and TNF- $\alpha^{15}$ in response to microbial pattern recognition receptor stimulation, which can attract and instruct other immune cells. Although PMN play a major role in the defense against pathogens and in inflammatory processes, Gasser and Schifferli ${ }^{11}$ demonstrated that PMNderived ectosomes block the inflammatory response in HMDM by inhibiting the release of TNF- $\alpha$, reducing the release of IL- 8 and inducing an immediate release of TGF- $\beta 1$, which is a known antiinflammatory cytokine. In contrast, other studies suggest the opposite, proposing that microvesicles released by PMN induce a pro-inflammatory effect. ${ }^{37}$ Mesri and Altieri ${ }^{37}$ analyzed a mixture of microvesicles released by PMN after a very long incubation time, one at which PMN undergo apoptosis or necrosis and release many intracellular fragments, which might have contained proinflammatory stimuli.

Using TEM, we characterized the morphology of MTB-induced PMN-derived ectosomes. They appear as ecto-organelles surrounded by membrane. The diameter of the ectosomes generated 
during the first hours of MTB infection assays ranged from 20 to $100 \mathrm{~nm}$, the same range previously described for ectosomes induced by $\mathrm{N}$-formylmethionyl-leucyl-phenylalanine (fMLP), ${ }^{23}$ ionomycin $^{30}$ and MTB. ${ }^{38}$ Apoptosis produces cell fragments appearing as vesicles, called apoptotic bodies, that phagocytic cells are able to engulf and quickly remove before the contents of the cell can spill out and damage nearby cells. ${ }^{39}$ It is noteworthy that the diameter of apoptotic bodies is greater than $400 \mathrm{~nm},{ }^{33}$ which is at least four times larger than the maximal MTB-induced ectosomes observed in our experiments. While phosphatidylserine is exposed on apoptotic bodies, it is also found on the surface of ectosomes, ${ }^{40}$ which allows them to be rapidly and selectively engulfed by phagocytes, ${ }^{41}$ presumably preventing inflammation.

In 2010, Gonzalez-Cano et al. $^{38}$ published the first paper on MTB-induced PMN-derived ectosomes, showing confocal microscope images of neutrophils infected with live Escherichia coli, Staphylococcus aureus and Leishmania mexicana and MTB. After $10 \mathrm{~min}$, all pathogens induced the release of similar microvesicles, both in terms of size and morphology. Thus far, few studies have been published on ectosome characteristics.

PMN-derived ectosomes stained poorly with PKH26. As suggested by Thom et al., ${ }^{42}$ it is possible that the partial staining arose
B
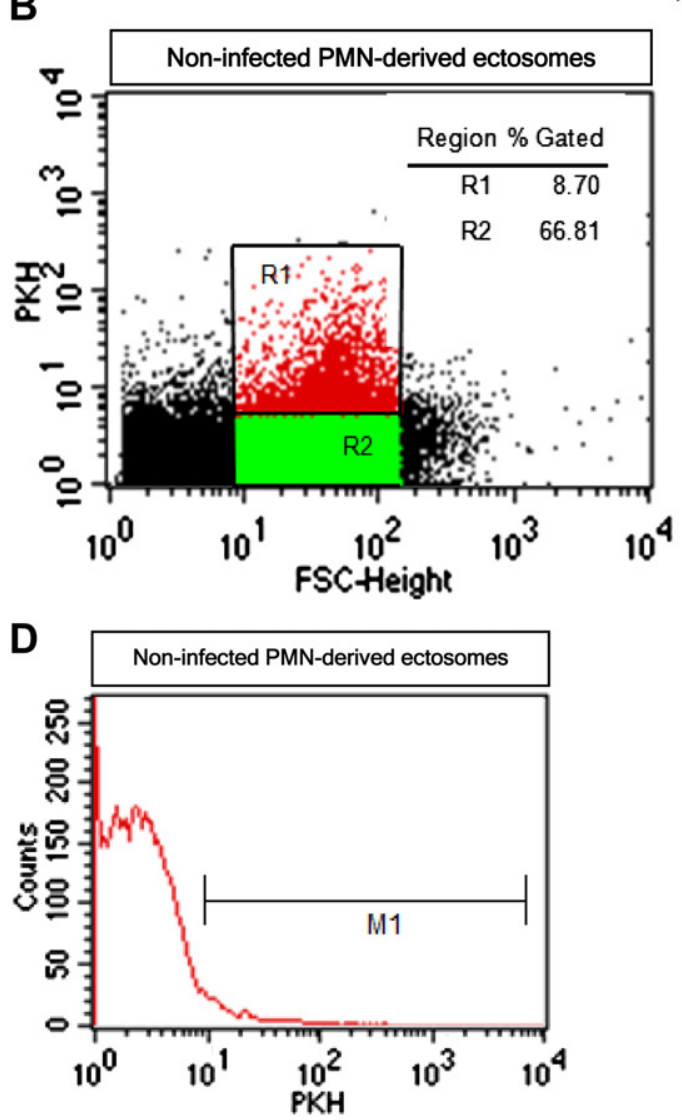
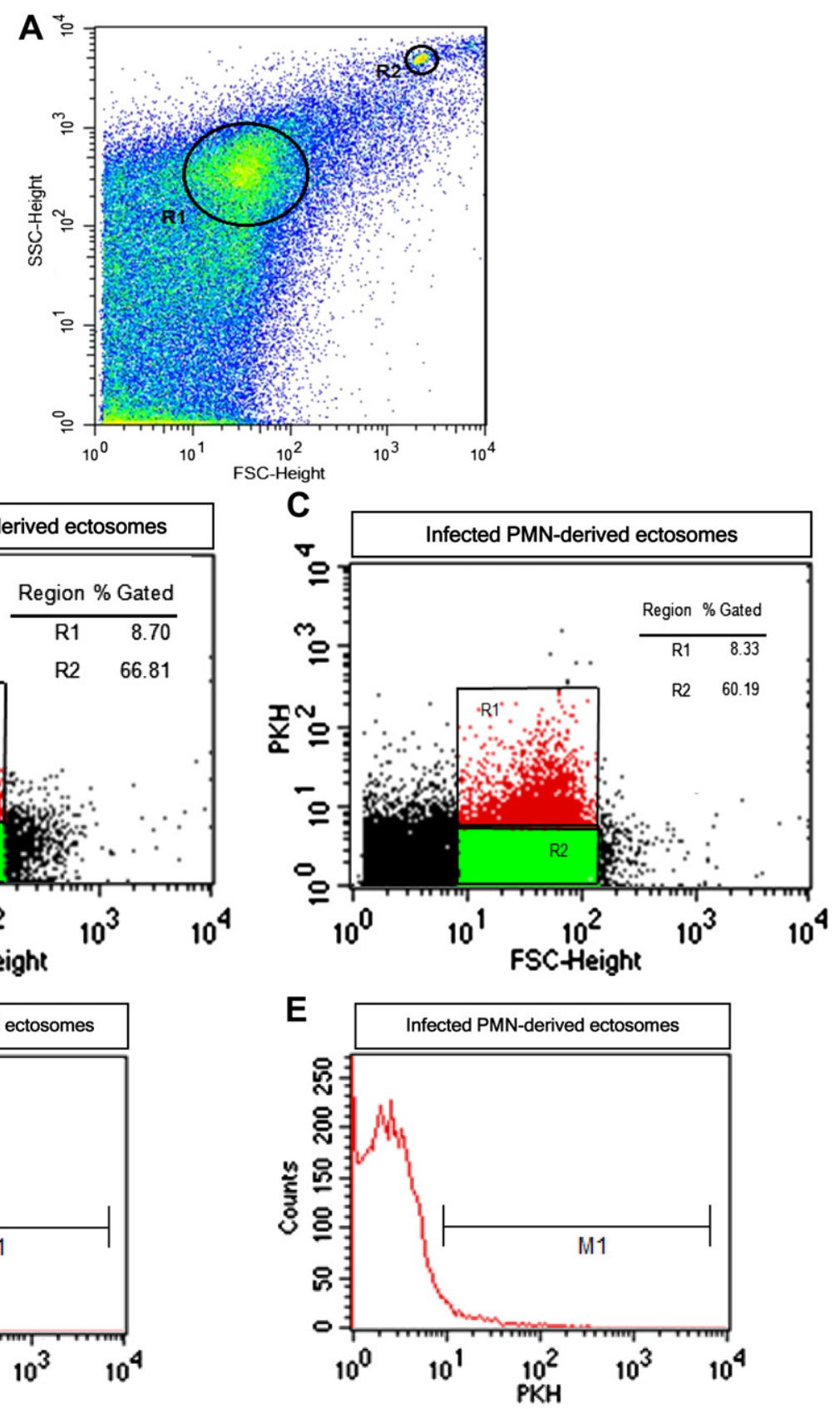

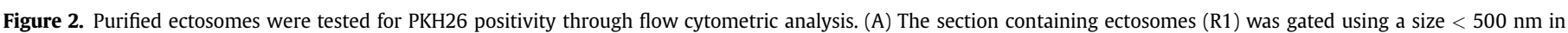

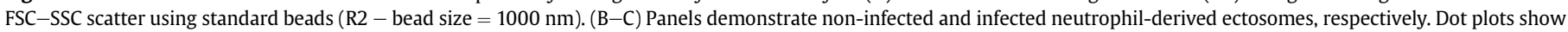

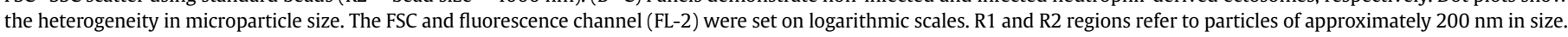

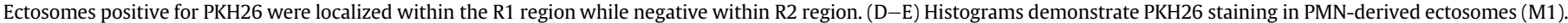

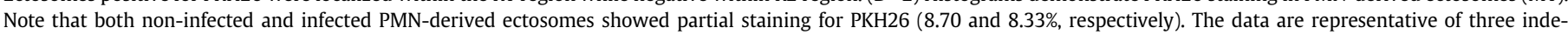
pendent preparations. 

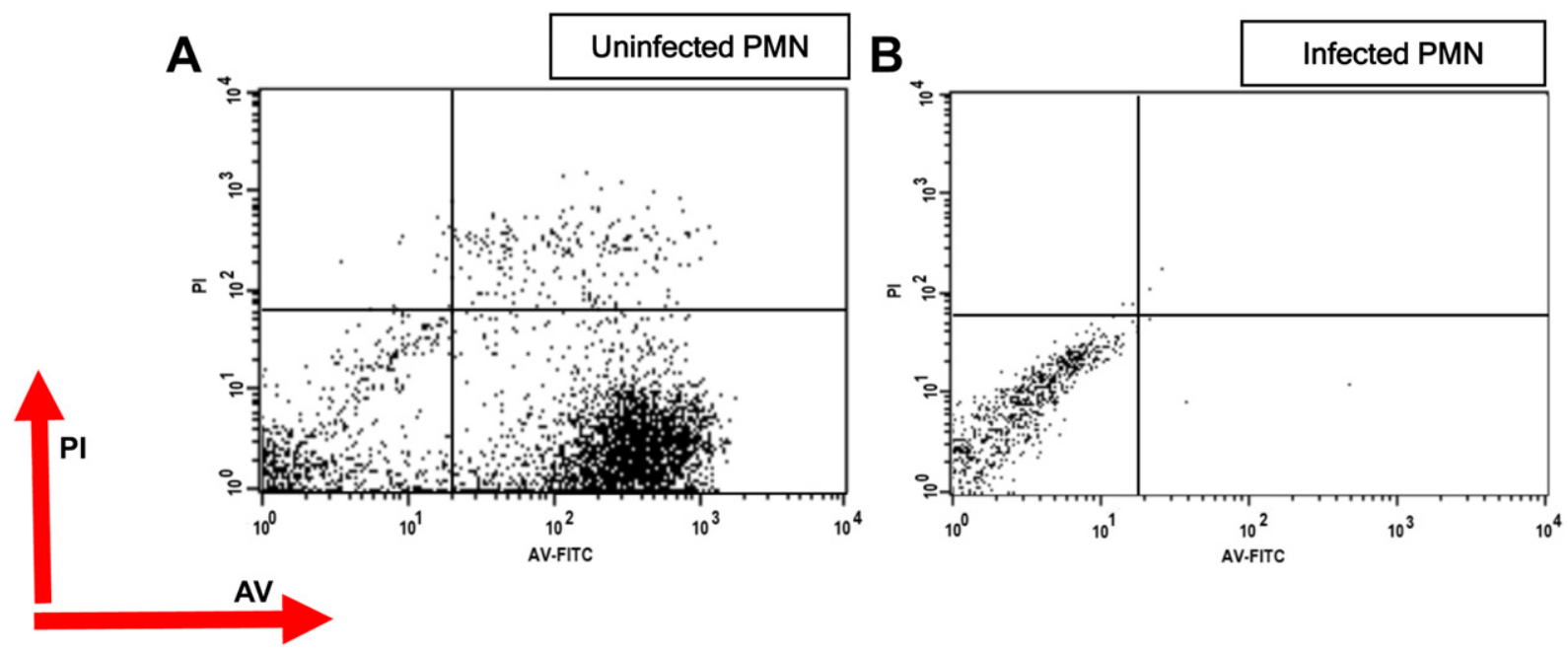

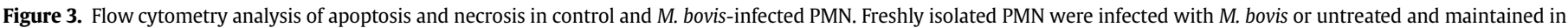

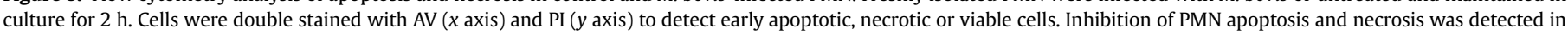
infected PMN. AV, annexin V; PI, propidium iodide.

because small vesicles ( $<300 \mathrm{~nm})$ may exhibit a smaller variety of surface markers than parent cells.

We tested whether apoptosis was occurring in the infected PMN by staining cells with annexin $\mathrm{V}$, which binds to surface phosphatidylserine and propidium iodide, which is retained in late apoptotic cells. ${ }^{34}$ As shown in Figure 3, M. bovis-infected neutrophils stained negative for both annexin $\mathrm{V}$ and propidium iodide $\left(1.0 \% \mathrm{AV}^{-} / \mathrm{PI}^{-}\right)$. However, uninfected neutrophils appeared to be undergoing apoptosis $\left(50.7 \% \mathrm{AV}^{+} / \mathrm{PI}^{-}\right)$. Mycobacterial infection of PMN induces increased expression of several function-associated surface molecules and upregulates mRNA for a set of cytokines and chemokines, including IL- $1 \alpha, \mathrm{IL}-1 \beta$ and MIP- $1 \beta,{ }^{34}$ as well as cytokine and chemokine receptors, such as IL-2R $\gamma$, IL-10R $\alpha$ and IL6R. Furthermore, spontaneous apoptosis of activated neutrophil granulocytes is inhibited by both $M$. bovis bacillus Calmette-Guerin $^{43}$ and MTB. ${ }^{44}$ In our experiment, MTB-induced PMN ectosomes were visualized and their secretion was enhanced upon activation but not apoptosis. These microvesicles were defined as ectosomes, in contrast to apoptotic bodies, by their morphological characteristics and by the viability of the neutrophils from which they were derived (Figure 3 ).

Previous studies have shown that PMN-derived ectosomes can modulate immunity and lead to an anti-inflammatory response. $11,27,45$ Our study is the first to demonstrate that MTBinduced PMN-derived ectosomes interfere with the antibacterial activity of human macrophages against virulent MTB, suggesting their importance in the immune processes, particularly in the antiTB immune response.

Major gaps remain in our knowledge of microvesicle pathophysiology and it is currently difficult to predict the biological consequences of PMN ectosome release in tissues due to their varied effects, with reported pro-inflammatory ${ }^{46}$ and anti-inflammatory effects on monocytes and macrophages. ${ }^{11,27}$ Mesri and Altieri ${ }^{37}$ found that ectosomes were associated with pro-coagulant and proinflammatory effects. Watanabe et al. $^{46}$ described neutrophilderived microparticles as being important pro-inflammatory agents containing platelet-activation factor (PAF), which led to the formation of platelet-leukocyte aggregates when the cells were present together. The release of PAF from stimulated PMN is a molecular mechanism with the potential to propagate inflammatory events. Trans-cellular activation by platelet and leukocyte microparticles is a well-established paradigm. ${ }^{37,46}$
Although ectosomes lead to mainly pro-coagulant and proinflammatory events in the circulation, they might be antiinflammatory or immunosuppressive in local tissue environments. The PMN enhancement of inflammation through the release of multiple mediators and enzymes has been extensively studied, but the mechanisms responsible for controlling this reaction are less well understood. The anti-inflammatory and immunosuppressive properties of PMN-derived ectosomes are novel findings, suggesting that they are active players in the regulation of inflammation.

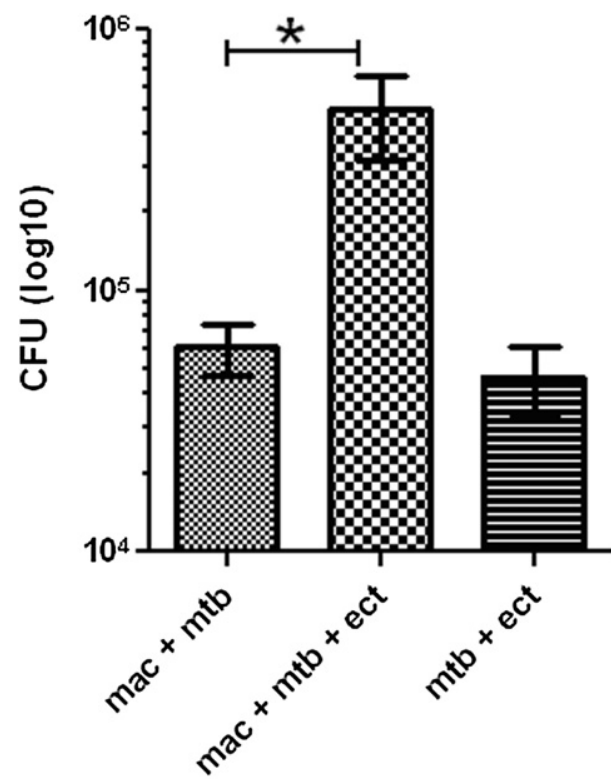

Figure 4. Influence of PMN-derived ectosomes on macrophage antimycobacterial activity. Infected PMN-derived ectosomes were added to cultures of MTB-infected macrophages. HMDM were infected with MTB at an infection ratio of $1: 1$ and seeded at a density of $10^{6} \mathrm{cell} /$ well. The mean CFU number at day 0 was $10^{6}$ for all conditions. The samples were incubated for $96 \mathrm{~h}$. At $96 \mathrm{~h}$ post-infection, the cells were permeabilized with $10 \%$ saponin and the bacterial suspensions were collected, sonically dispersed, serially diluted and plated in triplicate on 7H10 agar plates. CFU were cultured and counted after 6 weeks. The data represent the average number of $\mathrm{CFU} \pm$ standard error of the means (SEM) from independent experiments. The bars represent the experiments, which were performed in triplicate. *Statistically significant differences ( $P$ 0.0287) 
Communication is a major aspect in cell biology and occurs through both direct contacts between cells and through soluble substances that react with cells. Gasser and Schifferli ${ }^{11}$ found that PMN-derived ectosomes induced the release of TGF- $\beta 1$ by HMDM in vitro and were able to block the inflammatory response of macrophages through down-regulation of IL-8, IL-10 and TNF$\alpha$ secretion. Ectosome-to-cell contact was sufficient for this immunomodulatory function.

The concept that microvesicles released by some cells might influence other cells is rather new but probably represents a basic mechanism for delivering a message between cells in a highly efficient manner. The ability of PMN-derived ectosomes to downregulate the activity of macrophages may be relevant for other inflammatory processes or infectious diseases and further investigation in this field should be pursued.

Ongoing studies to extend the knowledge of neutrophilreleased ectosomes and their functional consequences may reveal novel insights into the mechanisms of cell-to-cell signaling, especially those by which ectosomes "reprogram" macrophages during acute inflammation and may also improve our understanding of the pathophysiology of MTB infection.

\section{Acknowledgments}

We acknowledge financial support from the CPqGM-FIOCRUZ, CAPES, CNPq and the Post Graduate Program in Clinical Medical of the UFRJ. We are thankful to INCQS for providing the strains of mycobacteria. We greatly appreciate the input of Theolis Barbosa Bessa (CPqGM-FIOCRUZ) for advice on experimental design, statistical analysis and interpretation.

We would also like to thank Thayna Meirelles Santos, Cláudio Filgueiras and Jorge Clarêncio Souza Andrade for excellent technical assistance.

\section{Funding: None.}

Competing interests: None declared.

\section{Ethical approval: Not required.}

\section{References}

1. van Crevel R, Ottenhoff TH, van der Meer JW. Innate immunity to Mycobacterium tuberculosis. Clin Microbiol Rev 2002;15:294-309.

2. Boechat N, Bouchonnet F, Bonay M, Grodet A, Pelicic V, Gicquel B, et al. Culture at high density improves the ability of human macrophages to control mycobacterial growth. J Immunol 2001;166:6203-11.

3. Di Liberto D, Caccamo N, Meraviglia S, Guggino G, La Manna MP, Sireci G, et al. Tuning inflammation in tuberculosis: the role of decoy receptors. Microbes Infect 2009;11:821-7.

4. Mehta PK, Karls RK, White EH, Ades EW, Quinn FD. Entry and intracellular replication of Mycobacterium tuberculosis in cultured human microvascular endothelial cells. Microb Pathog 2006;41:119-24.

5. Danelishvili L, McGarvey J, Li YJ, Bermudez LE. Mycobacterium tuberculosis infection causes different levels of apoptosis and necrosis in human macrophages and alveolar epithelial cells. Cell Microbiol 2003;5:649-60.

6. Appelberg R. Mycobacterial infection primes T cells and macrophages for enhanced recruitment of neutrophils. J Leukoc Biol 1992;51:472-7.

7. Lasco TM, Turner OC, Cassone L, Sugawara I, Yamada H, McMurray DN, et al. Rapid accumulation of eosinophils in lung lesions in guinea pigs infected with Mycobacterium tuberculosis. Infect Immun 2004;72:1147-9.

8. Eruslanov EB, Lyadova IV, Kondratieva TK, Majorov KB, Scheglov IV, Orlova MO, et al. Neutrophil responses to Mycobacterium tuberculosis infection in genetically susceptible and resistant mice. Infect Immun 2005;73:1744-53.

9. Shalekoff S, Tiemessen CT, Gray CM, Martin DJ. Depressed phagocytosis and oxidative burst in polymorphonuclear leukocytes from individuals with pulmonary tuberculosis with or without human immunodeficiency virus type 1 infection. Clin Diagn Lab Immunol 1998;5:41-4.

10. Aleman M, Garcia A, Saab MA, De La Barrera SS, Finiasz M, Abbate E, et al. Mycobacterium tuberculosis-induced activation accelerates apoptosis in peripheral blood neutrophils from patients with active tuberculosis. Am J Respir Cell Mol Biol 2002;27:583-92.
11. Gasser O, Schifferli JA. Activated polymorphonuclear neutrophils disseminate anti-inflammatory microparticles by ectocytosis. Blood 2004;104:2543-8.

12. Pellme S, Morgelin M, Tapper H, Mellqvist UH, Dahlgren C, Karlsson A. Localization of human neutrophil interleukin-8 (CXCL-8) to organelle(s) distinct from the classical granules and secretory vesicles. J Leukoc Biol 2006;79:564-73.

13. Luster $\mathrm{AD}$. The role of chemokines in linking innate and adaptive immunity. Curr Opin Immunol 2002;14:129-35.

14. Kobayashi M, Tsuda Y, Yoshida T, Takeuchi D, Utsunomiya T, Takahashi H, et al. Bacterial sepsis and chemokines. Curr Drug Targets 2006;7:119-34.

15. Korbel DS, Schneider BE, Schaible UE. Innate immunity in tuberculosis: myths and truth. Microbes Infect 2008;10:995-1004.

16. Territo MC, Ganz T, Selsted ME, Lehrer R. Monocyte-chemotactic activity of defensins from human neutrophils. J Clin Invest 1989;84:2017-20.

17. Aleman $M$, Beigier-Bompadre $M$, Borghetti $C$, de la Barrera $S$, Abbate $E$, Isturiz M, et al. Activation of peripheral blood neutrophils from patients with active advanced tuberculosis. Clin Immunol 2001;100:87-95.

18. Fiorenza G, Farroni MA, Bogue C, Selenscig D, Lamas DM, Dlugovitzky D. Functional characteristics of neutrophils and mononuclear cells from tuberculosis patients stimulated in vitro with heat killed M. tuberculosis. Arch Med Res 2007;38:526-33.

19. Pluskota E, Woody NM, Szpak D, Ballantyne CM, Soloviev DA, Simon DI, et al. Expression, activation, and function of integrin alphaMbeta2 (Mac-1) on neutrophil-derived microparticles. Blood 2008;112:2327-35.

20. Nolan S, Dixon R, Norman K, Hellewell P, Ridger V. Nitric oxide regulates neutrophil migration through microparticle formation. Am $J$ Pathol 2008;172:265-73.

21. Cocucci E, Racchetti G, Meldolesi J. Shedding microvesicles: artefacts no more Trends Cell Biol 2009;19:43-51.

22. Pilzer D, Gasser O, Moskovich O, Schifferli JA, Fishelson Z. Emission of membrane vesicles: roles in complement resistance, immunity and cancer. Springer Semin Immunopathol 2005;27:375-87.

23. Gasser O, Hess C, Miot S, Deon C, Sanchez JC, Schifferli JA. Characterization and properties of ectosomes released by human polymorphonuclear neutrophils. Exp Cell Res 2003;285:243-57.

24. Stein JM, Luzio JP. Ectocytosis caused by sublytic autologous complement attack on human neutrophils. The sorting of endogenous plasma-membrane proteins and lipids into shed vesicles. Biochem J 1991;274:381-6.

25. Pfeffer SR. Two rabs for exosome release. Nat Cell Biol 2010;12:3-4.

26. Sadallah S, Eken C, Schifferli JA. Ectosomes as immunomodulators of inflammation and immunity. Clin Exp Immunol 2011;163:26-32.

27. Eken C, Gasser O, Zenhaeusern G, Oehri I, Hess C, Schifferli JA. Polymorphonuclear neutrophil-derived ectosomes interfere with the maturation of monocyte-derived dendritic cells. J Immunol 2008;180:817-24.

28. Distler JH, Huber LC, Hueber AJ, Reich 3rd CF, Gay S, Distler O, et al. The release of microparticles by apoptotic cells and their effects on macrophages. Apoptosis 2005;10:731-41.

29. Andrews RK, Berndt MC. Microparticles facilitate neutrophil/platelet crosstalk. Blood 2008;112:2174-5.

30. Hess C, Sadallah S, Hefti A, Landmann R, Schifferli JA. Ectosomes released by human neutrophils are specialized functional units. $J$ Immunol 1999;163:4564-73.

31. Qu Y, Dubyak GR. P2X7 receptors regulate multiple types of membrane trafficking responses and non-classical secretion pathways. Purinergic Signal 2009;5:163-73.

32. Abdul-Majid KB, Ly LH, Converse PJ, Geiman DE, McMurray DN, Bishai WR. Altered cellular infiltration and cytokine levels during early Mycobacterium tuberculosis sigC mutant infection are associated with late-stage disease attenuation and milder immunopathology in mice. BMC Microbiol 2008;8:151.

33. Ozaki T, Nakahira S, Tani K, Ogushi F, Yasuoka S, Ogura T. Differential cell analysis in bronchoalveolar lavage fluid from pulmonary lesions of patients with tuberculosis. Chest 1992;102:54-9.

34. Vermes I, Haanen C, Steffens-Nakken H, Reutelingsperger C. A novel assay for apoptosis. Flow cytometric detection of phosphatidylserine expression on early apoptotic cells using fluorescein labelled Annexin V. J Immunol Methods 1995;184:39-51.

35. Tan BH, Meinken C, Bastian M, Bruns H, Legaspi A, Ochoa MT, et al. Macrophages acquire neutrophil granules for antimicrobial activity against intracellular pathogens. J Immunol 2006;177:1864-71.

36. Eum SY, Kong JH, Hong MS, Lee YJ, Kim JH, Hwang SH, et al. Neutrophils are the predominant infected phagocytic cells in the airways of patients with active pulmonary TB. Chest 2010;137:122-8.

37. Mesri M, Altieri DC. Endothelial cell activation by leukocyte microparticles. J Immunol 1998;161:4382-7.

38. Gonzalez-Cano P, Mondragon-Flores R, Sanchez-Torres LE, Gonzalez-Pozos S, Silva-Miranda M, Monroy-Ostria A, et al. Mycobacterium tuberculosis H37Rv induces ectosome release in human polymorphonuclear neutrophils. Tuberculosis 2010;90:125-34.

39. Savill J. Recognition and phagocytosis of cells undergoing apoptosis. $\mathrm{Br} \mathrm{Med}$ Bull 1997;53:491-508.

40. Dalli J, Norling LV, Renshaw D, Cooper D, Leung KY, Perretti M. Annexin 1 mediates the rapid anti-inflammatory effects of neutrophil-derived microparticles. Blood 2008;112:2512-9. 
41. Wu Y, Tibrewal N, Birge RB. Phosphatidylserine recognition by phagocytes: a view to a kill. Trends Cell Biol 2006;16:189-97.

42. Thom SR, Yang M, Bhopale VM, Huang S, Milovanova TN. Microparticles initiate decompression-induced neutrophil activation and subsequent vascular injuries. J Appl Physiol 2010;110:340-51.

43. Suttmann H, Lehan N, Bohle A, Brandau S. Stimulation of neutrophil granulocytes with Mycobacterium bovis bacillus Calmette-Guerin induces changes in phenotype and gene expression and inhibits spontaneous apoptosis. Infect Immun 2003;71:4647-56.
44. Blomgran R, Desvignes L, Briken V, Ernest JD. Mycobacterium tuberculosis inhibits neutrophil apoptosis, leading to delayed activation of naïve CD4 T cell. Cell Host Microbe 2012;11:81-90.

45. Thery C, Ostrowski M, Segura E. Membrane vesicles as conveyors of immune response. Nat Rev Immunol 2009;9:581-93.

46. Watanabe JJ, Marathe GK, Neilsen PO, Weyrich AS, Harrison KA, Murphy RC et al. Endotoxins stimulate neutrophil adhesion followed by synthesis and release of platelet-activating factor in microparticles. J Biol Chem 2003;278:33161-8. 